D I C $\mathbf{E}$ latindex @ideAs Econpapers ODialnet ÎnDICEs MIAR Sucupira

\title{
PROYECTO DE INVESTIGACIÓN SOBRE LA ACTUALIZACIÓN PEDAGÓGICA DEL PROFESORADO EN EDUCACIÓN SECUNDARIA OBLIGATORIA
}

\author{
Beatriz Martínez García \\ Psicóloga y Orientadora Educativa. Universidad de Murcia. \\ beatrizmartinezizpi@gmail.coml \\ Esther Martínez Catillo \\ Psicóloga y Orientadora Educativa. Universidad de Murcia. \\ esthermartinezcastillotin1@gmail.com \\ Raquel Carayol Dengra
}

Pedagoga y Orientadora Educativa. Universidad de Murcia.

raquelcarayol@gmail.com

Para citar este artículo puede utilizar el siguiente formato:

Beatriz Martínez García, Esther Martínez Catillo y Raquel Carayol Dengra (2021): "Proyecto de investigación sobre la actualización pedagógica del profesorado en educación secundaria obligatoria", Revista DELOS, (Especial noviembre 2021). En línea:

https://doi.org/10.51896/DELOS/NYJO7595

\section{RESUMEN}

En este trabajo se presenta una propuesta de investigación acerca de la formación continua del docente. El objetivo principal de este estudio es conocer si el profesorado se implica de manera activa en su formación continua, conociendo aspectos como la presencia o ausencia de dicha formación, la edad, el género, utilización de TICs, así como la opinión de diferentes agentes educativos. Para ello, se establece una muestra representativa, perteneciente a cuatro centros educativos, seleccionados de manera aleatoria. Para la obtención de la información, se han creado tres instrumentos, dirigidos cada uno de ellos a los grupos que componen nuestra muestra: alumnos, docentes y equipo directivo. Esta propuesta, se presenta como un estudio innovador, que permitiría conocer cómo es la formación continua del docente, las preferencias de los mismos, así como la importancia de mantener una actualización continua en la labor docente dentro del aula.

Palabras clave: Formación continua, nuevas tecnologías, actualización de los conocimientos, docente, estudiante.

\section{RESEARCH PROJECT ON PEDAGOGICAL UPDATING OF TEACHERS IN COMPULSORY SECONDARY EDUCATION}




\section{ABSTRACT}

This paper presents a research proposal on teacher in-service training. The main objective of this study is to know if teachers are actively involved in their continuous training, knowing aspects such as the presence or absence of such training, age, gender, use of ICTs, as well as the opinion of different educational agents. For this purpose, a representative sample was established, belonging to four randomly selected educational centers. To obtain the information, three instruments have been created, each of them addressed to the groups that make up our sample: students, teachers and management team. This proposal is presented as an innovative study, which would allow us to know how the continuous training of teachers, their preferences, as well as the importance of maintaining a continuous updating in the teaching work in the classroom.

Keywords: Continuous learning, new technologies, updating knowledge, teacher, student.

\section{INTRODUCCIÓN}

A lo largo de este proyecto se va a estudiar la implicación que tiene el profesorado en la Educación Secundaria Obligatoria en su formación continua. Para ello, se van a analizar diferentes variables que pueden intervenir en esta formación y cómo puede afectar dicha implicación en la comunidad educativa.

La formación que reciben los docentes a lo largo de su carrera universitaria resulta incompleta para conseguir una educación eficaz y de calidad, debido a los múltiples cambios inmersos en la sociedad de la actualidad lo que desencadena la necesidad de trabajar en una actualización continua del equipo docente para desempeñar sus funciones de forma eficaz (López et al., 2018). La formación continua del profesorado da la posibilidad de que los docentes adquieran diferentes aprendizajes que puedan llevarlos a la práctica con el fin de una mejora en la calidad de la enseñanza, tanto en la experiencia escolar como en los aprendizajes que desarrolle el alumnado (González y Cutanda, 2017).

Cuando los docentes reciben una actualización pedagógica se logra una adaptación, una flexibilidad y una creatividad de los conocimientos y de las habilidades que se van adquiriendo a través de distintas situaciones, incrementando sus competencias a lo largo de su vida (Escudero et al., 2017). Por ello, en la actualidad, es fundamental la adquisición de una formación continua e integral por parte del profesorado para inculcar todo lo mencionado con anterioridad a las nuevas generaciones (López et al., 2018). Esta formación presenta una valoración limitada puesto que, los docentes no reciben una preparación pedagógica específica, para comprender y hacer frente a las diferentes situaciones que se pueden dar en el entorno escolar, ni antes ni durante su labor como docente (Escudero, 2017).

Por todo lo mencionado anteriormente, se va a realizar un estudio cuasicuantitativo, puesto que para la evaluación de la investigación se cuenta con instrumentos tanto cualitativos como 
cuantitativos, con el fin de conocer cuál es el nivel de implicación que presentan los docentes de Educación Secundaria Obligatoria en relación con la formación continua.

\section{Conceptualización de la formación continua docente}

El concepto de formación continua docente hace referencia a toda actividad que realicen los docentes con la finalidad de desencadenar un ejercicio de adquisición, renovación y ampliación de sus propios conocimientos, competencias y habilidades, por tanto, todo este proceso de renovación y reciclaje debe ir encaminado hacia el espíritu crítico, la investigación y autonomía del propio docente para no estancarse en la rutina y monotonía diaria, desencadenando frustración y desmotivación laboral (López et al., 2018). Debido a los cambios en los que se encuentra sumergida la sociedad actualmente, es de especial relevancia que los docentes adquieran competencias profesionales adaptadas a las demandas de la sociedad que permitan obtener calidad en la enseñanza y aprendizaje, por tanto, el reciclaje del profesorado con relación a su formación hace posible que estos se adapten de forma eficaz (Iglesias et al., 2018).

Según el MEFP (2019), respecto a la formación que reciben los docentes, en la LOE, se relega a las administraciones educativas la planificación de diversas actividades que deben asegurar la participación, diversidad y el acceso gratuito al profesorado para poder llevar a cabo una formación continuada. La formación continua del profesorado puede dividirse en dos modelos, según entiendan la formación del docente según centrada en el proceso y contenido de la formación (formación como entrenamiento técnico individual) o según la manera en la que puede ser utilizada dicha información (formación como desarrollo profesional colegiado), siendo este último modelo de un carácter más específico respecto al contexto del trabajo (Alfageme y Nieto, 2017). En España se ofrecen cinco modalidades, las cuales se incluyen en los modelos mencionados, para la formación continua de los docentes: cursos impartidos por especialistas, seminarios impartidos por entidades organizativas o los propios docentes, grupos de trabajo para la elaboración de materias curriculares, proyectos de formación en el centro educativo de referencia, así como congresos destinados a un tema determinado (MEFP, 2019).

\section{Influencia de la formación continua del profesorado en el proceso de enseñanza y aprendizaje}

La formación continua del profesorado, debido a la poca importancia que se ha puesto sobre la misma, carece de una organización sistemática que permita elaborar y diseñar programas destinados a esta (León y Latas, 2005). En los últimos años, el proceso de enseñanza-aprendizaje ha sufrido un cambio, donde la implicación activa del alumnado en su propia formación se ha antepuesto como una necesidad dentro del aula, por lo que, debido a este motivo, se puede intuir la necesidad de cambiar la docencia, así como las metodologías de enseñanza tradicionales (Romero et al., 2017). 


\section{Importancia de las TIC en la formación continua del profesorado en la actualidad}

La formación pedagógica del profesorado relacionada con las TIC y su incorporación en la práctica educativa es escasa, es por esto por lo que, la mayoría de docentes reconocen que precisan de una mejor formación acerca de las mismas (Lores et al., 2019). Ante los cambios producidos en la actualidad, el Instituto Nacional de Tecnologías Educativas y de Formación del Profesorado (INTEF), ha estandarizado la competencia digital con el fin de desarrollar en los docentes las destrezas necesarias para llevar a cabo su labor profesional de forma eficaz (Pozo et al., 2020). Considerando la gran problemática que ha ocasionado la pandemia (SARSCoV-2) en el ámbito de la educación, se hace necesario enfocar la importancia de las nuevas tecnologías en una enseñanza virtual con el fin de garantizar la continuidad de la enseñanza y pensar nuevas formas de trabajar en el aula con el alumnado, lo que conlleva, a abordar la formación continua del docente a esta nueva perspectiva (Dussel, 2020). A lo largo de la formación continua, es necesario que los docentes adquieran conocimientos y relacionados con la integración de las herramientas digitales en las aulas y su puesta en práctica, con la finalidad de incluirlas en el proceso de enseñanza-aprendizaje para que el alumnado adquiera competencias básicas relacionadas con este aspecto, y de esta manera, favorecer una educación de calidad dentro de la innovación (Iglesias et al., 2018).

\section{OBJETIVOS}

El objetivo general de este proyecto de investigación es conocer si el profesorado de Educación Secundaria Obligatoria se implica de forma activa en su Actualización Pedagógica.

Por otro lado, los objetivos específicos serán:

- Conocer el porcentaje de los docentes que realizan una formación continua.

- Analizar cómo influye la edad y el género en la formación continua del docente.

- Conocer si en la actualización pedagógica de los docentes intervienen las nuevas tecnologías.

- Conocer qué métodos utilizan los docentes para la formación continua.

- Estudiar el grado de implicación en la formación continua según la función que el docente desempeña en el centro.

- Valorar la percepción que presenta el alumnado acerca de la formación continua del profesorado.

- Valorar la percepción que presenta el equipo directivo acerca de la formación continua del profesorado. 


\section{METODOLOGÍA}

\section{Descripción de la población y la muestra: participantes}

Esta investigación se va a realizar en cuatro centros educativos de Educación Secundaria Obligatoria de carácter público en la ciudad de Murcia. La elección de estos ha sido en base a las siguientes características contextuales y sociodemográficas: nivel cultural medio de las familias, nivel socioeconómico medio-alto, nivel de estudios de los familiares medio, centros recreativos y culturales próximos al entorno de cada uno de los centros y número de estudiantes superior a 350 en cada uno de los centros. La población de esta investigación se divide en tres grupos: docentes, equipo directivo y alumnado, todos ellos pertenecientes a los cuatro centros seleccionados. Respecto a los docentes, la población constará de 245 docentes, de los cuales, con un margen de error del $5 \%$ y un nivel de confianza del 95\%, serán necesarios 180 docentes para que la muestra sea representativa. Por otro lado, la población de alumnado de los centros escogidos es de 1894, con un margen de error del $5 \%$ y un nivel de confianza del $95 \%$, serán necesarios 384 alumnos/as para que la muestra sea representativa. Por último, relacionado con el equipo directivo, será necesaria la participación del director y jefe de estudios de cada uno de los equipos directivos de estos centros para poder obtener una muestra representativa con un margen de error del $5 \%$ y un nivel de confianza del $95 \%$. La estrategia de muestreo de esta investigación se realizará de manera no probabilística a través de voluntarios. A las muestras representativas de cada uno de los grupos, se le ha añadido un $20 \%$ de participantes para solventar posibles casos de muerte muestral. Con la selección de los diferentes grupos, se pretende recabar y contrastar la información ofrecida por los mismos, quedando reflejada la triangulación dentro de la investigación.

\section{Descripción de las variables}

La variable dependiente de esta investigación es la formación continua del profesorado. Por otro lado, se van a encontrar una serie de variables independientes, las cuales serían:

La presencia o ausencia de formación continua

La edad

El género

Nuevas tecnologías

Métodos utilizados para la formación continua

Percepción del alumnado

Percepción del Equipo Directivo

Descripción de los materiales y/o instrumentos 
Para la valoración de la formación continua por parte de los docentes, en primer lugar, se analizarán unos datos sociodemográficos que serán el sexo; la edad; años trabajados como docentes; puesto que desempeña en el centro; y cuál es el método de formación continua que más utilizan, para la respuesta de esta información aparecerán cuatro opciones para marcar la o las que más se adecúen a la postura que cada docente. Posteriormente a estos datos, y en el mismo Anexo se presentará una escala de actitudes, en concreto la aditiva de Likert, dirigida a los docentes, en la que aparecerán ítems relacionados con su propia formación continua. Esta escala es caracterizada por tener tanto ítems positivos como ítems negativos, y a través de la regla de la simetría los ítems negativos se convierten en positivos para realizar la suma total. Para las respuestas a las diferentes cuestiones aparecerá una escala numerada del 1 al 5 , para que los docentes que realicen esta escala marquen aquella opción que crean más conveniente (véase en Anexo I). Por otro lado, para conocer la valoración del alumnado respecto a la formación continua del profesorado, se administrará un cuestionario diferencial semántico de Osgood, en el cual los alumnos deberán valorar, del 1 al 5 , la formación del profesorado y cómo influye esta en su aprendizaje a través de los adjetivos bipolares que se presentan (Anexo II). Por último, se realizará un grupo de discusión a los equipos directivos, en el cuál, participarán el Director y el Jefe de Estudios de cada uno de los cuatros centros educativos elegidos para la población de la investigación, siendo un total de ocho participantes. Con el grupo de discusión se pretende establecer una interacción entre los participantes mediante un debate sobre unas cuestiones planteadas, con el fin de entrevistar al grupo y conseguir una visión de consenso de una temática determinada (Barbour, 2013). Dicho debate será grabado con el consentimiento de los participantes para luego poder analizarlo. Se van a proponer una serie de cuestiones (Anexo III) que serán trabajadas en el grupo de discusión, y después se analizará el debate que se haya llevado a cabo para poder interpretar los resultados que se obtengan.

\section{METODOLOGÍA}

\section{Descripción del método y el procedimiento}

Para establecer el punto de partida de esta propuesta de investigación, en primer lugar, en el momento inicial de la investigación, entre las componentes del grupo se estableció un debate para elegir la temática que se iba a desarrollar en la investigación, eligiendo por consenso la formación continua del profesorado en la etapa de Educación Secundaria Obligatoria, estableciendo el problema del estudio y el objetivo general que iba a abordar. Seguidamente, se llevó a cabo una revisión bibliográfica acerca de los contenidos que engloba esta investigación, tanto los que se van a estudiar como aquellos que van a influir en la misma. Este proceso de búsqueda bibliográfica tuvo una duración de una semana. Una vez seleccionados aquellos artículos y documentos complementarios pertinentes para la investigación, se comenzó a redactar la introducción y fundamentación teórica plasmando aquellos conceptos relevantes acorde con la temática y se concretaron los objetivos específicos, los cuales permiten alcanzar el objetivo general propuesto, empezando la fase de desarrollo de la investigación. Una vez concretada la fundamentación teórica y los objetivos de nuestra propuesta, procedimos al desarrollo de la metodología, comenzando por la 
selección de la muestra y el proceso mediante el cual iba a ser seleccionada la misma. La muestra propuesta se considera representativa, puesto que ha sido calculada mediante una calculadora muestral e incluye tres grupos diferentes dentro de la comunidad educativa: alumnado, docentes y equipo directivo. Posteriormente, se establecieron las variables de nuestro estudio, identificando la variable dependiente y las variables que influyen en la misma. De esta manera, y teniendo como base dichas variables, la metodología de nuestra investigación y la muestra seleccionada, fueron creados los instrumentos que servirían para recoger la información, siendo tres instrumentos de recogida de información. Por último, en la fase de momento final de la investigación, se ha llevado a cabo el desarrollo de la estimación del éxito del proyecto justificando la viabilidad y la utilidad que presenta.

\section{Estimación del éxito del proyecto}

La investigación propuesta nace de la necesidad de conocer la formación continua por parte del profesorado en la etapa de Educación Secundaria Obligatoria ya que esta formación influye en la mejora del proceso de enseñanza y aprendizaje tanto para el alumnado como para el propio docente. Es importante la actuación continua de conocimientos, habilidades, estrategias y metodologías por parte de los docentes para abordar las demandas que se encuentran actualmente dentro del aula. Este proyecto es realizado para solventar las cuestiones anteriormente mencionadas, y para ello se han tenido en cuenta diferentes factores que hacen que la propuesta sea viable. En primer lugar, debido a la importancia que tiene la formación continua, se ha considerado relevante tener en cuenta la percepción no solo de los docentes, sino también del alumnado y del equipo directivo de cada uno de los centros que se ha seleccionado. Por otro lado, cabe destacar, que la muestra propuesta es representativa a la vez que accesible, lo que facilita la realización de la investigación. Los instrumentos elaborados son de fácil comprensión y administración, además de que no exigen una formación específica por parte del administrador a la hora de presentar las instrucciones del instrumento y llevarlo a cabo. Es por esto que, puede ser administrado en diferentes formatos, tanto en modalidad presencial como en modalidad online, sin necesidad de invertir una cantidad de horas elevadas. Otro punto fuerte que destacar es la financiación de la propuesta, puesto que los recursos económicos necesarios serían mínimos, esto permite la replicabilidad de la propuesta en un futuro, por parte de otros investigadores. Una de las utilidades que se pueden derivar de esta propuesta es la de poder invertir en la formación del profesorado de manera acorde a las preferencias de los participantes, lo cual derivaría en una mayor implicación del docente en su propia actualización. La formación continua docente frecuentemente adopta un plano secundario dentro de su actuación, por lo que se considera otro aspecto destacable de esta propuesta es la visibilidad e impacto que puede llegar a tener al resto de las instituciones educativas. Relacionado con lo anterior, gracias a esta investigación los docentes pueden llegar a reflexionar, y ser autocríticos acerca de la importancia de su propia formación, puesto que, en muchos casos, no son conscientes de la ausencia de esta en su labor docente. 


\section{REFERENCIAS}

Alfageme, M. B., y Nieto, J. M. (2017). Los docentes de la enseñanza obligatoria en España y las actividades de formación continua. Perfiles educativos, 39(158), 148-165.

Barbour, R. (2013). Los grupos de discusión en investigación cualitativa. Ediciones Morata.

Dussel, I. (2020). La formación docente y los desafíos de la pandemia. Revista Científica EFI. DGES 6(10), 13-25.

Escudero, J. M., López, M. T. C., y Alonso, J. F. T. (2017). APRENDIZAJE DOCENTE Y DESARROLLO PROFESIONAL DEL PROFESORADO. Profesorado. Revista de Currículum y formación de profesorado, 21(3), 83-102.

Escudero, J. M. (2017). La formación continua del profesorado de la educación obligatoria en el contexto español. Profesorado. Revista de Currículum y Formación de Profesorado, 21(3), 1-20. https://www.redalyc.org/pdf/567/56752489001.pdf

González, M. T. G., y López, M. T. C. (2017). La formación continuada del profesorado de enseñanza obligatoria: incidencia en la práctica docente y el aprendizaje de los estudiantes. Profesorado. Revista de Currículum y Formación de Profesorado, 21(3), 103-122.

Iglesias, M. J., Lozano, I., y Roldán, I. (2018). La calidad e innovación educativa en la formación continua docente: un estudio cualitativo en dos centros educativos. Revista Iberoamericana de Educación, 77(1), 13-34.

León, B., y Latas, C. (2005). Nuevas exigencias en el proceso de enseñanza aprendizaje del profesor universitario en el contexto de la convergencia europea: La formación en técnicas de aprendizaje cooperativo. REIFOP, 8(6).

Ley Orgánica 2/2006, de 3 de mayo, de Educación. Boletín Oficial del Estado, 106, de 4 de mayo de 2006. https://www.boe.es/buscar/pdf/2006/BOE-A-2006-7899-consolidado.pdf

López, J., Moreno, A. J., y Pozo, S. (2018). Influencia del género y la edad en la formación continua multidisciplinar de los docentes de cooperativas de enseñanza. INNOVA Research Journal 3(8.1), 42-59. https://doi.org/10.33890/innova.v3.n8.1.2018.756

Lores, B., Sánchez, P., y García, M. R. (2019). La formación de la competencia digital en los docentes. Profesorado, Revista de Currículum y formación del profesorado, 23(4), 234-260. http:// doi.org/10.30827/profesorado.v23i4.11720. 
Ministerio de Educación y Formación Profesional (MEFP). (2019). Formación permanente del profesorado en las administraciones educativas. http://www.educacionyfp.gob.es/mc/redieeurydice/sistemas-educativos/profesorado/formacion-permanenteprofesorado.htm|\#: :text=La\%20formaci\%C3\%B3n\%20permanente\%20del\%20profesorado \%2C\%20t\%C3\%A9cnica\%2C\%20did\%C3\%A1ctica\%20y\%20profesional.\&text=Se\%20con sideran\%20un\%20derecho\%20y\%20un\%20deber\%20de\%20todos\%20los\%20docentes.

Pozo, S., López, B., Fernández, M., y López, J. A. (2020). Análisis correlacional de los factores incidentes en el nivel de competencia digital del profesorado. Revista Electrónica Interuniversitaria de Formación del Profesorado 23(1), 143-159.

Romero, R., Castejón, F. J., López, V. M., y Fraile, A. (2017). Evaluación formativa, competencias comunicativas y TIC en la formación del profesorado. Comunicar: Revista Científica de Comunicación y Educación, 25(52), 73-82. https://doi.org/10.3916/C52-2017-07 